http://dx.doi.org/10.18778/0208-6107.32.10

\author{
Leon Miodoński \\ (iD) https://orcid.org/0000-0001-8525-3813 \\ Institut für Philosophie \\ Universität Breslau \\ leon.miodonski@uwr.edu.pl
}

\title{
WEISHEIT BEI SCHOPENHAUER UND DIE FRAGE IHRER AKTUALITÄT
}

\begin{abstract}
Zusammenfassung
Schopenhauers Weisheitsbegriff wurde aus zwei verschiedenen Perspektiven analysiert. Erstens, im Kontext der frühen Philosophie, wo „das bessere Bewusstsein“ eine Schlüsselrolle spielte. Der Höhepunkt dieser Zeit waren das Werk Die Welt als Wille und Vorstellung und radikal formulierte philosophische Exklusivität. Zweitens, im Kontext von Essays-Sammlung Parerga und Paralipomena, und besonders einem der Essays Aphorismen zur Lebensweisheit, wo sich Schopenhauer vom Metaphysiker und Buddhisten in den Moralisten und den Lehrer vervandelte, der die Verfahrensregeln in der irrationalen Welt des Willens zeigte, so dass das Leid die geringste Teilnahme an der realen Existenz des Menschen hatte. Diese Akzentverschiebung erfolgte vor allem dank der gründlichen und tiefen Lektüre von Baltasar Gracians Das Hand-Orakel und Kunst der Weltklugheit. Gracian lehrte aber an keiner Stelle, wie man sich von der Welt befreien kann, sondern eher wie man in einer Welt des Bösen, voll von Gegensätzen, Intrigen und Niederträchtigkeiten leben kann.
\end{abstract}

\section{Schlüsselwörter:}

Arthur Schopenhauer, Weisheit, Baltasar Gracian, deutsche Philosophie

Der Begriff der Weisheit hat sich in die Tradition eigentlich aller Zeiten und Kulturen eingeschrieben und ist immer dort präsent, wo die Besinnung über die Welt verschiedene Formen der Lebensweisheit, des praktischen Wissens, des Mythos oder der Philosophie annahm. Der Begriff änderte sich so, wie sich das menschliche Bewusstsein sowie die Fähigkeit, theoretisch-begriffliche Modelle zu schaffen, sublimierte und änderte.

Im Falle Schopenhauers haben wir mit einem philosophischen Moment zu tun, den man als eine Übergangsphase betrachten kann, und zwar von dem theoretisch-spekulativen Denken bis zur sozial-politischen Praxis, von dem nachkantianischen Idealismus, der bis in die 30er Jahre des 19. Jahrhunderts 
vorherrschte, bis zu den positivistischen und empirischen Systemen, die sich in den 30er Jahren und später entwickelten und die sich auf die europäische Philosophie immer deutlicher auswirkten. Konkret ausgedrückt: Schopenhauer, der im Jahre 1788 geboren wurde, reifte intellektuell unter dem Einfluss der Romantiker und der Vorlesungen von Schleiermacher und Fichte; er starb im Jahre 1860, als sich niemand mehr mit den idealistischen Systemen ernsthaft beschäftigte. Diese Verschiebung des philosophischen Schwerpunktes lässt sich beispielhaft am Schopenhauerschen Verständnis der Weisheit nachvollziehen, die er anfangs als „wahre Philosophie“ allgemein bezeichnete, also als Erfassen der Ganzheit; und er versuchte dabei, ihr eigentliches Wesen, d. h. „wahre Weisheit" zu verstehen. ${ }^{1}$ Später, abgesehen von dem höheren, metaphysischethischen Standpunkt, neigte er in seinen Essays zum Begriff der „Lebensweisheit“, die er als „die Kunst [verstand], das Leben möglichst angenehm und glücklich durchzuführen." ${ }^{\text {2 }}$ Allerdings verdankte Schopenhauer seinen Ruf überwiegend seinen Essays und den kleinen ethischen Abhandlungen.

Zunächst ist ein wichtiger Aspekt hervorzuheben, der die Persönlichkeit und die Haltung Schopenhauers beeinflusste und der im Kontext des Weisheitsbegriffes interessant ist, der aber einer psychopathologischen Fachuntersuchung bedürfte, und zwar sein eigenes Leben, seine Unfähigkeit, psychologische Probleme zu bewältigen: seine toxische Familie, Arroganz, seine Neigung zur Depression, sein cholerischer Charakter. All diese Elemente schaffen ein Bündel von Gegensätzen, aus denen aber eine solide philosophische Reflexion resultiert. Es scheint, als könnte man die Regeln aus ,dem HandOrakel und (der) Kunst der Weltklugheit" von Baltazar Gracian auf Schopenhauer anwenden: „Kenntniß seiner selbst, an Sinnenart, an Geist, an Urtheil, an Neigungen”, d. h. „keiner kann Herr über sich seyn, wenn er sich zuvor begriffen hat" ${ }^{\text {"3 }}$, und „seine Lieblingsfehler kennen“, d.h. „eine kühle Selbstüberwindung. “4

Das Vorwort zur ersten Auflage von Die Welt als Wille und Vorstellung beginnt mit folgenden Worten: „Wie dieses Buch zu lesen sei, um möglicherweise verstanden werden zu können, habe ich hier anzugeben mir vorgesetzt. - Was durch dasselbe mitgetheilt werden soll, ist ein einziger Gedanke. Dennoch konnte ich, aller Bemühungen ungeachtet, keinen kürzeren

\footnotetext{
${ }^{1}$ Arthur Schopenhauer, „Die Welt als Wille und Vorstellung,” Bd. I, in Arthur Schopenhauer, Werke in fünf Bänden, Hrsg. von Ludger Lütkehaus (Zürich: Hoffmans Verlag, 1994), 186.

${ }^{2}$ Arthur Schopenhauer, „Parerga und Paralipomena," Bd. IV, in Arthur Schopenhauer, Werke in fünf Bänden, hg. von Ludger Lütkehaus (Zürich: Hoffmans Verlag, 1994), 313.

${ }_{3}$ Baltasar Gracian, Das Hand-Orakel und Kunst der Weltklugheit, aus dem Spanisch übers. von Arthur Schopenhauer, in Arthur Schopenhauer, Der handschriftliche Nachlaß, Bd. IV 2, Hg. von Arthur Hübscher (Frankfurt am Main: Kramer, 1975), 171.

${ }^{4}$ Ibid., 202-203.
} 
Weg ihn mitzutheilen finden, als dieses ganze Buch. - Ich halte jenen Gedanken für Dasjenige, was man unter dem Namen der Philosophie sehr lange gesucht hat, und dessen Auffindung, eben daher, von den historisch Gebildeten für so unmöglich gehalten wird, wie die des Steines der Weisen [...]. Je nachdem man jenen einen mitzutheilenden Gedanken von verschiedenen Seiten betrachtet, zeigt er sich als Das, was man Metaphysik, Das, was man Ethik und Das, was man Aesthetik genannt hat; und freilich müßte er auch dieses alles seyn, wenn er wäre, wofür ich ihn, wie schon eingestanden, halte. “5

Diese Worte scheinen ein Schlüssel zu sein, sein Werk als ein philosophisches Manifest zu lesen, in der Zeit, in der man viele ähnliche Manifeste vorfindet. Die Welt als Wille und Vorstellung, die dem Geiste und dem Paradigma des deutschen Idealismus entwuchs, bildet zugleich dessen Destruktion: die Kritik des Begriffes des Absoluten, die Ablehnung der Begriffe „Gott" und „Seele“, die Kritik der Geschichte und der Rationalität der geschichtlichen Entwicklung, das Konzept der empirisch begründeten Metaphysik usw. Was wäre also die Weisheit, wenn wir die Standardauffassungen der neuzeitlichen, christozentrisch orientierten Philosophie ablehnen würden?

Schopenhauer formte das Verstehen der Philosophie und das Ziel des philosophischen Denkens gründlich um und stützte sich dabei auf das Modell der indischen Philosophie, auf den Übergang von Samsara zu Nirwana, - „die uralte Weisheit der Inder", die erlaubte, den Schleier der Maja durchzudringen. ${ }^{6}$

In den Schopenhauerschen Begriffen drückt sich das im Übergang von der „Bejahung des Willens zum Leben“ zu der „Verneinung des Willens zum Leben“ aus. In dieser Auffassung gehört Weisheit zum Nirwana-Zustand, d. h. zur „Verneinung des Willens zum Leben“, sie hat dann aber keinen diskursiven und begrifflichen Charakter, sie bedeutet vielmehr den Bewusstsein-Zustand: „das bessere Bewußtsein”.

Der Begriff „das bessere Bewußtsein” ist ein interessantes Konstrukt der frühen Reifeperiode Schopenhauers. Lange vor der Konzeptualisierung seines Lebenswerkes, bereits in den Manuskripten aus dem Jahre 1812, hat er das grundsätzliche Weisheitsmotiv herausgearbeitet, das dann zum Leitmotiv seiner Philosophie geworden ist: ,in dieser Zeitlichen, Sinnlichen, Verständlichen Welt gibt es wohl Persönlichkeit und Kausalität, ja sie sind sogar nothwendig. - Aber das bessre Bewußtseyn in mir erhebt mich in eine Welt, wo es weder Persönlichkeit und Kausalität noch Subjekt und Objekt mehr gibt. Meine Hoffnung und Mein Glaube ist daß dieses bessre (übersinnliche außerzeitliche) Bewußtseyn mein einziges werden wird."

\footnotetext{
${ }^{5}$ Schopenhauer, „Die Welt,” Bd. I, 7.

${ }^{6}$ Ibid., 37.

${ }^{7}$ Schopenhauer, Der handschriftliche Nachlaß, Bd. I, 42.
} 
Die aus vier Büchern bestehende Konstruktion „Die Welt als Wille und Vorstellung" bezieht sich auf das Hinaufsteigen der Weisheitsleiter, die der Mensch durch die Vernunft und die Welterfahrung, durch die abstrakten Begriffe und die Ideen, die er erzeugt, erreichen kann. Der wahre Sinn der Erkenntnis, d. h. die „wahre Philosophie“, wie es Schopenhauer bezeichnet - mit anderen Worten: die höchste Stufe der Weisheit, die man erreichen kann - objektiviert sich erst am Ende und beruht auf dem Hinausgehen über die Begrifflichkeit der Philosophie. Es kommt dann zum Akte der höchsten Erkenntnis, zur Harmonie zwischen dem Subjekt und dem Objekt, zur moralischen Vollkommenheit. Die „wahre Philosophie“ beruht auch auf der Bestimmung des radikalen, kognitiven Elitarismus: sie steht im Gegensatz zur gewöhnlichen Erkenntnis, deren Subjekt ,der gewöhnliche Mensch” als „Fabrikware der Natur” ist, der nicht imstande ist, über die Bejahung der Gegenständlichkeit, die höhere Erkenntnis und die Erkenntnis des Genies hinauszugehen, deren Sinn gerade auf der Ablehnung der Gegenständlichkeit beruht. $^{8}$

„Weisheit und Genie, diese zwei Gipfel des Parnassus menschlicher Erkenntniß, wurzeln nicht im abstrakten, diskursiven, sondern im anschauenden Vermögen. Die eigentliche Weisheit ist etwas Intuitives, nicht etwas Abstraktes. Sie besteht nicht in Sätzen und Gedanken, die Einer als Resultate fremder oder eigener Forschung im Kopfe fertig herumtrüge: sondern sie ist die ganze Art, wie sich die Welt in seinem Kopfe darstellt." “9

Die höchste Weisheit bedeutet also einen Übergang zur vollen Erkenntnis des Wesens der Welt, der Welt des Willens, d.h. der Welt des Bösen. Erfordert werden dabei die außerordentlichen Veranlagungen - wie bereits erwähnt - die überhaupt keinen Zusammenhang mit der Fähigkeit haben, die abstrakten Begriffe der Philosophie zu verwenden. Die wahre Erkenntnis gibt also die Möglichkeit „der Aufhebung des Wollens, der Erlösung durch Freiheit, der Ueberwindung und Vernichtung der Welt." ${ }^{10}$ Es kommt zur Ablehnung der Welt, solch einer Welt, die uns in der empirischen Erfahrung erscheint. Schopenhauer bezeichnet das oft mittels der quasi religiösen Begriffe als „die Erlösung von der Welt" ${ }^{\text {"11 }}$ oder „die Erlösung aus dem Jammer dieser Welt." ${ }^{\text {”2 }} \mathrm{Er}$ bezieht sich oft auf die christliche Mystik, Quietismus und die Askese (Meister Eckhart, Angelus Silesius, Jeane Marie de la Mothe-Guyon u.a.), wo er „Anleitung zum unmittelbaren Innenwerden Dessen [bemerkte], wohin weder Anschauung noch Begriff also überhaupt keine Erkenntniß reicht." ${ }^{\text {13 }}$

\footnotetext{
${ }^{8}$ Schopenhauer, „Die Welt,” Bd. I, $255 \mathrm{ff}$.

${ }^{9}$ Ibid., Bd. II, 88.

${ }^{10}$ Ibid., Bd. I, 429.

${ }^{11}$ Ibid., Bd. I, 215.

12 Ibid., Bd. I, 424.

${ }^{13}$ Ibid., Bd. II, $710 \mathrm{ff}$.
} 
Eine Schlüsselbedeutung haben die letzten Worte in Die Welt als Wille und Vorstellung: „was nach gänzlicher Aufhebung des Willens übrig bleibt, ist für alle Die, welche noch des Willens voll sind, allerdings Nichts. Aber auch umgekehrt ist Denen, in welchen der Wille sich gewendet und verneint hat, diese unsere so sehr reale Welt mit allen ihren Sonnen und Milchstraßen - Nichts." ${ }^{\text {14 }}$

Dieser Ausschnitt hat eine besondere Bedeutung. Zusammengefasst wurden hier das Wesen der Philosophie Schopenhauers, wie auch der grundsätzliche Punkt des Angriffes all jener Kritiker, die ihn als Nihilisten bezeichneten, der alles Dasein und Existenz in Frage stelle, oder als Irrationalisten, der über die Gesetze der Vernunft und der Logik hinausgehe. ${ }^{15}$ Das Schopenhauersche „Nichts” ist keine Nichtigkeit, kein Fehlen, sondern eine andere Art des Daseins ohne Täuschung und ein Zustand des Quietismus. Dieses „Nichts“ ist „das PradschnaParamita der Buddhaisten, das »Jenseits aller Erkenntnisß«, d. h. der Punkt, wo Subjekt und Objekt nicht mehr sind." ${ }^{.16}$ Im philosophischen Diskurs der damaligen Epoche war das die Formeln, die, welche eher unklar und schwer zum Aneignen für den europäischen Leser waren, weil das Verstehen der Weisheit des buddhistischen Gedankens sich erst in der Anfangsphase der philologischen Rezeption befand. In der Tat war Schopenhauer einer der Wenigen, die den Geist dieser Philosophie intuitiv verstanden, auch wenn sie über die durchaus bescheidenen Quellen verfügten.

Das in Die Welt als Wille und Vorstellung von 1819 dargestellte Konzept der Philosophie als die Negation der Welt kann man als eine philosophische Provokation des jungen, radikalen, sich seines Wertes selbstbewussten und streitsüchtigen Intellektuellen betrachten, eine Provokation, die gegen die anerkannten und populären Systeme des deutschen Idealismus gerichtet war. Dieses Werk - interessant und originell - hatte einen grundsätzlichen Nachteil, d. h. es zeichnete sich durch die rein intellektuelle Auffassung aus, die die Postulate vom regulativen Charakter formulierte, während im Buddhismus es um die Praxis geht, um die Suche nach der Weisheit im Prozess der Umgestaltung des menschlichen Bewusstseins, in der Beziehung des Menschen zur Welt. ${ }^{17}$

\footnotetext{
${ }^{14}$ Ibid., Bd. I, 528.

${ }^{15}$ Siehe: Ernst Bergmann, Die Erlösungslehre Schopenhauers (München: Rösl \& Cie, 1921). „Ich empfinde Schopenhauers Asketismus als eine romantische Verirrung, die nur dadurch gemildet wird, daß sie platonisch bleibt”, 63; „,Für die Gegenwart jedenfalls bedeutet das Schopenhauersche Heiligenideal schlimmste Kulturabsage”, 65.

${ }^{16}$ Arthur Schopenhauer, Sämtliche Werke, Bd. 2, Nach der ersten von Julius Frauenstädt besorgten Gesamtausgabe neu bearb. und hrsg. von Arthur Hübscher, 4. Aufl. durchges. von Angelika Hübscher, (Mannheim: Brockhaus, 1988), 621. (Die Welt als Wille und Vorstellung, Bd. 2).

${ }^{17}$ Vgl. zu diesem Thema: Dalai Lama, Die Essenz der Lehre Buddhas, aus dem Engl. übers. von Jochen Lehner, (München: Heyne, 2014); Michael von Brück, Einführung in den Buddhismus (Berlin: Insel Verlag, 2011).
} 
Schopenhauer war sich dieser Schwäche sicherlich bewusst. In seinen weiteren Texten setzte er zwar das allgemeine, im ersten Band Die Welt als Wille und Vorstellung formulierte Konzept fort, aber er knüpfte nicht mehr an die scharf formulierte Prozedur der Ablehnung der Welt an, die den ersten Band abschließt. Arthur Hübscher, der Klassiker der Schopenhauerforschung, nahm diese Änderung als den Abschied vom Pessimismus aus Die Welt als Wille und Vorstellung wahr und als Umkehr in die Richtung der praktischen Philosophie Parerga und Paralipomena. ${ }^{18}$

Mit dem Verlauf der Zeit verwandelte sich Schopenhauer vom Metaphysiker und Buddhisten in den Moralisten und den Lehrer, der die Verfahrensregeln in der irrationalen Welt des Willens zeigte, so dass das Leid die geringste Teilnahme an der realen Existenz des Menschen hatte. Diese Akzentverschiebung erfolgte vor allem dank der gründlichen und tiefen Lektüre von Baltasar Gracian, dem spanischen Jesuiten aus dem 17. Jahrhundert. Unter seinem Einfluss stand Schopenhauer seit der zweiten Hälfte der 20er-Jahre. ${ }^{19}$ Die ruhige Lebensweisheit von Gracian, die auf der Besinnung und der moralischen Selbstvervollkommung beruhte, findet ihre besondere Wiederspiegelung in der Sammlung der Essays Parerga und Paralipomena, und besonders in einem der Essays Aphorismen zur Lebensweisheit.

Um die Werke von Gracian - wie auch von Calderon, Cervantes, Lope - lesen zu können, begann Schopenhauer seit dem Jahre 1825 Spanisch zu lernen. Als Endeffekt entstand die Übersetzung ins Deutsche einer von zahlreichen Arbeiten Gracians, und zwar Oráculo manual y arte de prudencia (1647) - Das Hand-Orakel und Kunst der Weltklugheit. Es ist ein Buch, das sich bis heute einer großen Beliebtheit erfreut und das geschieht nicht ohne großen Anteil Schopenhauers. An dieser Stelle empfiehlt es sich, eine interessante Begebenheit zu erwähnen. Im Jahre 1992 wurden fast 200000 Exemplare von Das Hand-Orakel in der englischen Übersetzung verkauft. ${ }^{20}$ Das Buch hielt sich 18 Wochen lang auf der Bestsellersliste Washington Post, die ersten beiden Wochen sogar durchgängig an erster Stelle. ${ }^{21}$

Die Weisheit Gracians war auf eine besondere Art und Weise damit verbunden, wozu Schopenhauer selbst in seiner Reflexion über die Welt kam. Die dreihunderte und letzte Regel aus dem „Hand-Orakel“ -,,Mit einem Wort, ein

\footnotetext{
${ }^{18}$ Arthur Hübscher, Denker gegen den Strom. Schopenhauer: Gestern - Heute - Morgen (Bonn: Bouvier, 1973), 152.

19 Siehe: Schopenhauers Brief an Brockhaus vom 15. Mai 1829, in Arthur Schopenhauer, Gesammelte Briefe, 2. Aufl, hrsg. von A. Hübscher (Bonn: Bouvier, 1987), 111-113.

${ }^{20}$ Siehe: Baltasar Gracian, The Art of Worldly Wisdom. A Pocket Oracle, trans. by Christopher Maurer (New York: Doubleday, 1992).

${ }^{21}$ Juan Domínguez Lasierra, „El año en que Gracián fue best-seller en los USA,” Turia. Revista cultural 54 (2000): 149-154. Vgl. auch: Johanna Schumm, „Zur Wiederkehr der Verstellung. Die gegenwärtige Rezeption von Graciáns Oráculo manual als Ratgeber," Komparatistik Online 1 (2014): 204-228.
} 
Heiliger (zu) sein “22 - enthält das Motiv des Heiligen aus den letzten Abschnitten des ersten Bandes von „Die Welt als Wille und Vorstellung“. Ausdrücke wie „ein fruchtbares Genie“, „ein tiefer Verstand“ oder „ein erhabener Geschmack“ weisen auf eine sprachliche Nähe zu Schopenhauer hin. Gracian lehrte aber an keiner Stelle, wie man sich von der Welt befreien kann, sondern eher wie man in einer Welt des Bösen, voll von Gegensätzen, Intrigen und Niederträchtigkeiten leben kann. Die Quintessenz seiner Weisheit bildet die Tugendlehre:

„Die Tugend ist das gemeinsame Band aller Vollkommenheiten, und der Mittelpunkt aller Glückseligkeit. Sie macht einen Man vernünftig, umsichtig klug, verständig weise, tapfer, überlegt, redlich, glücklich, beifällig, wahrhaft und zu einem Helden in jedem Betracht. Drei Dinge, welche im Spanischen mit einem S anfangen, machen glücklich: Heiligkeit, Gesundheit und Weisheit. [...] [Tugend] allein ist sich selbst genug: sie macht den Menschen im Leben liebenswürdig und im Tode denkwürdig. “23

Die Zeit der Arbeit an der Übersetzung von Das Hand-Orakel fällt auf dieselbe Zeit, als Schopenhauer das Manuskript Eristische Dialektik vorbereitete. Beide Abhandlungen erschienen erst nach dem Tod des Philosophen. Und beide bilden gleichzeitig in gewissem Sinne einen Wendepunkt - es entwickelt sich „der andere Schopenhauer", der seine Willensphilosophie durch zahlreiche Bezüge auf die Weltliteratur und die ganze philosophische Tradition stärkt und damit ein gelehrsames Werk schafft, das die universelle Weisheit wiederspiegelt. Dabei unterliegt er einer Transformation in die Richtung der Haltung eines Moralisten und Lebenslehrers. ${ }^{24}$ Selbstverständlich würde Schopenhauer mit dieser Ansicht nicht übereinstimmen und auf der Kohärenz seines Konzeptes beharren. Dies drückt er im Vorwort zu den Aphorismen zur Lebensweisheit aus, wo er deutlich betont, dass er in seinen Essays von einem höheren, metaphysisch-ethischen Standpunkt absieht, der das Wesen seiner Philosophie ausmacht. ${ }^{25}$

Auf der anderen Seite erinnerte der Lebensstil Schopenhauers keineswegs an die asketische Suche nach der Weisheit, in der Kasteiung und in der Ablehnung von allem irdischen Vergnügen, von dem wir im vierten Buch von Die Welt als Wille und Vorstellung lesen können. Ganz im Gegenteil: die Zeit des reifes Alters und des Greisenalters in Frankfurt zeichnete sich durch die Stabilität eines reichen Bürgers aus, der das Vergnügen nicht vermied, das ihm Geld ermöglichte. Das bemerkte Karl Gutzkow bereits im Jahre 1862. Er beschrieb scherzhaft die Tagesordnung des „Frankfurter Asketen“. Der Bericht - ein bisschen geändert - befand sich später in der Abhandlung Arthur Schopenhauer.

\footnotetext{
${ }^{22}$ Gracian, Das Hand-Orakel und Kunst der Weltklugheit, 259.

${ }^{23}$ Ibid.

${ }^{24}$ Hübscher, Denker gegen den Strom, 153.

${ }^{25}$ Schopenhauer, Parerga, 313-314.
} 
Ein Wort der Verteidigung (1863) von Ernst Otto Lindner ${ }^{26}$ : „Wohnen in einer eleganten Etage. Aufstehen zwischen 7 und 8 Uhr. Der [...] Kaffee, die Pfeife. Das einladende weiche Sofa mit beneidendwerthen Luxus interessanter Lektüre, abwechselnd dann das Auge geschlossen und hinausgeschaut in die Ideenwelt. Die Feder ergriffen, die Wahrnehmung der Seele niedergeschrieben. [...] Keine lärmende Kinderumgebung [...], Keine Hausfrau, die über ihre Anschaffungen eine Rechnung bringt. [...] Vor Tisch «bläßt» der Asket selbst behaglich «eine halbe Stunde die Flöte». Nimmt die Besuche der Verehrer und Geschäftsfreunde entgegen und schlendert dann an die Table d'hôte des ersten Hotels in Frankfurt, [...] hier spannt sich der ermüdete Geist im leichten Gespräch aus. [...] Der fünfte oder sechste Gang der Speisen ist vorüber, jetzt wandelt der vom Weltschmerz gedrückte «Einsiedler» nach Hause, macht sich wieder seinen Kaffee, stopft wieder seine Pfeife von einem fünf $\mathrm{Fu}$ langen Weichselrohr, welche Länge allein den Rauch gehörig abkühle. Auf dem Sopha ausgestreckt, hat der Asket mit diesem allerdings unbequemen Rohr vielleicht einige «Mühe»; aber der Tisch liegt voll «leichter Lektüre»; [...] Putz, der Pudel, bellt; der Abend naht; der einsame fröhliche Spazierlauf um die Stadt beginnt. Das Theater ist geöffnet, die Oper bringt ein [...]. Der Glückliche, Sorgenlose geht in die Lesegesellschaft, liest die «Times», schlendert wieder in den Englischen Hof zum Nachtessen und zu «einer halben Flasche Wein» zurück; dann endlich sinkt er nach 10 Uhr sanft in Morpheus' Arme, die ihn fest umschlungen halten. Er genießt das Glück eines gesunden Schlafs. So ein Tag wie der andere. “27

In diesem satirischen Bild der ,praktischen” Askese, die Gutzkow spöttelnd als „Martyrium der Wahrheit” bezeichnet, verbirgt sich aber eine gewisse, kluge Haltung, die die Lebensweisheit des alten Schopenhauers charakterisiert. Diese Haltung wird in seinen Essays ausgedrückt und ist kompatibel mit den Hinweisen Gracians und anderer Moralisten: „ein ruhiger Blick aufs Ganze“28 - wie es Alfred Schmidt schön formuliert hat.

Es bleibt noch die Frage nach der Originalität der Schopenhauerschen Auffassung der Weisheit zu betrachten. Über die Aktualität des Gedankengutes Schopenhauers wurde schon viel geschrieben. ${ }^{29}$ Sein Einfluss auf die kulturelle Geschichte der letzten 150 Jahre steht außer Zweifel. Als einer der Wenigen nahm er damals die Bedeutung der Naturwissenschaften, der Psychologie, des

\footnotetext{
${ }^{26}$ Ernst Otto Lindner, Arthur Schopenhauer. Von ihm. Ueber ihn. Ein Wort der Vertheidigung und Julius Frauenstädt, Memorabilien, Briefe und Nachlassstücke (Berlin: A.W. Hayn, 1863), 21-23.

${ }^{27}$ Karl Gutzkow, „Arthur Schopenhauer's Lehre und Leben”, in Karl Gutzkow, Unterhaltungen am häuslichen Herd, dritte Folge, 2. Band, (Leipzig: Heinrich Brockhaus Verlagsort, 1862), 274.

${ }^{28}$ Alfred Schmidt, Die Wahrheit im Gewande der Lüge. Schopenhauers Religionsphilosophie (München, Zürich: Piper, 1986), 26.

${ }^{29}$ Siehe: Volker Spierling, Hrsg., Schopenhauer im Denken der Gegenwart. 23 Beiträge zu seiner Aktualität, hrsg. von V. Spierling (München, Zürich: Piper, 1987); Wolfgang Schirmacher, Hrsg., Schopenhauers Aktualität. Ein Philosoph wird neu Gelesen (Wien: Passagen Verlag, 1988).
} 
Unbewussten, der Sexualität wahr. Heute betont man besonders sein Engagement für den Schutz und die Rechte der Tiere, für die Ökologie wie auch für die modernen Neurowissenschaften und Neurophilosophie. ${ }^{30}$

Am Anfang der 70er-Jahre des 20. Jahrhunderts schrieb Ewald Bucher in der Abhandlung Schopenhauers Lebensweisheit heute (einer der wenigen Abhandlungen zu diesem Thema), dass ,die Grundlage seines Werks und seiner Weisheit die Wahrheit [ist] ${ }^{631}$, die nicht ewig und absolut gegeben ist, die aber eine ständige Suche und Aktualisierung erfordert. Man sollte damit übereinstimmen. Die Korrelation zwischen der Wahrheit und der Weisheit war ein Leitmotiv während des Höhepunktes des Interesses an der Philosophie Schopenhauers, also am Ende des 19. Jahrhunderts und am Anfang des 20. Jahrhunderts. Sein Werk wurde zum Sprachrohr der suggestiven Wahrheit, die er noch als ein junger Mann erfahren hat: die auf die metaphysische Weltnatur bezogene Wahrheit, die in dem berühmten, allgemein zitierten Fragment aus dem Manuskript Cholerabuch zum Ausdruck kommt: „In meinem 17 ten Jahre[,] ohne alle gelehrte Schulbildung, wurde ich vom Jammer des Lebens so ergriffen, wie Buddha in seiner Jugend, als er Krankheit, Alter, Schmerz und Tod erblickte. Die Wahrheit, welche laut und deutlich aus der Welt sprach, überwandt bald die auch mir eingeprägten Jüdischen Dogmen, und mein Resultat war, daß diese Welt kein Werk eines allgültigen Wesens seyn könnte, wohl aber das eines Teufels, der Geschöpfe ins Daseyn gerufen, um am Anblick ihrer Quaal sich zu weiden: darauf deuteten die Data, und der Glaube, daß es so sei, gewann die Oberhand.“32

Diese fundamentale Wahrheit - „Siehe da, das ist das Leben“33 - faszinierte vor allem Schriftsteller und Dichter. Sie waren weniger an der Kohärenz des philosophischen Systems Schopenhauers interessiert, sondern vielmehr an dem Wahrheitsgehalt und der Suggestivität der Schopenhauerschen Weltbetrachtung, die direkt auf die existenzielle Kondition des Menschen bezogen war. Sehr charakteristisch sind die Worte von Lew Tolstoj nach der Lektüre von „Parerga und Paralipomena“: „Ein unaufhörliches Entzücken über Schopenhauer und eine Reihe von geistigen Genüssen, wie ich sie nie gekostet habe. [...] Aber jetzt bin ich überzeugt, dass Schopenhauer der genialste Mensch ist." ${ }^{34}$ Ähnlich haben sich August Strindberg, Thomas Mann, André Gide und viele andere im ganz Europa ausgedrückt.

Heute ist Schopenhauer aufs Regalbrett unter dem Namen „Klassik“ geraten. Zur Zeit interessieren sich - von Fachleuten abgesehen - wenige für die

\footnotetext{
30 Dieter Birnbacher, „Schopenhauer und die moderne Neurophilosophie," Schopenhauer Jahrbuch 86 (2005): 133-148.

${ }^{31}$ Ewald Bucher, „Schopenhauers Lebensweisheit heute,” Schopenhauer Jahrbuch 55 (1974): 36.

${ }^{32}$ Schopenhauer, Der handschriftliche Nachlaß, Bd. IV 1, 96.

${ }^{33}$ Ibid., Bd. III, 391.

${ }^{34}$ Gerd Hoffmans, Hrsg., Über Arthur Schopenhauer, 2. Aufl. (Zürich: Diogenes, 1978), 189.
} 
philosophischen Streitigkeiten, die vor Jahrhunderten stattfanden, weil sie keineswegs kompatibel mit dem gegenwärtigen Weltbild sind, auch nicht für die Fragen der philosophischen Spekulationen. Deshalb beruht der Wert dessen, was man als „Lebensweisheit“ Schopenhauers bezeichnen kann, auf dessen zeitloser Aktualität, die der Leser verstehen und in Kontemplation genießen kann. Es ist die Wahrheit, die authentisch erfahren wird.

Wenn Schopenhauer die philosophischen Essays nicht geschrieben hätte, hätte er das Schicksal von Karl Christian Friedrich Krause oder Jakob Friedrich Fries geteilt - die großen Geister ihrer Zeit, die man schnell vergessen hat. Dank der Essays Schopenhauers können wir ihn in der Kontinuität einer bedeutenden philosophischen Tradition betrachten, von Michel de Montaigne, Francis Bacon, Blaise Pascal, John Milton bis zu Walter Benjamin und Stanislaw Lem. Als Anmerkung dazu lässt sich noch erwähnen, dass der in Polen meist gelesene Essay Schopenhauers Eristische Dialektik ist. In den letzten dutzend Jahren des 21. Jahrhunderts erschienen neun separate Ausgaben von diesem Essay, die schnell verkauft wurden.

Wenn ich eine persönliche Bemerkung am Ende machen dürfte, dann möchte ich Folgendes sagen. Aus meiner langjährigen Erfahrung als akademischer Lehrer resultiert, dass Schopenhauer - trotz des ziemlich geringen Interesse an Philosophie in Polen - zu der geringen Gruppe der Philosophen gehört, von denen die Studenten etwas wissen, mit denen sie sich gern beschäftigen; sie fragen während des Unterrichts nach Einzelheiten, schreiben Diplomarbeiten und manchmal auch Doktorarbeiten. Schopenhauer stößt auf Interesse der Studenten, wohl deshalb, weil er deren Achtsamkeit und Sensibilität berührt.

\section{BIBLIOGRAPHIE}

Bergmann, Ernst. Die Erlösungslehre Schopenhauers. München: Rösl \& Cie, 1921.

Birnbacher, Dieter. „Schopenhauer und die moderne Neurophilosophie.” Schopenhauer Jahrbuch 86 (2005): 133-148.

Brück, Michael von. Einführung in den Buddhismus. Berlin: Insel Verlag, 2011.

Bucher, Ewald. „Schopenhauers Lebensweisheit heute.” Schopenhauer Jahrbuch 55 (1974): 27-38.

Dalai Lama, Die Essenz der Lehre Buddhas. Aus dem Engl. übers. von Jochen Lehner. München: Heyne, 2014.

Gracian, Baltasar. Das Hand-Orakel und Kunst der Weltklugheit. Aus dem Spanisch übers. von Arthur Schopenhauer. In Arthur Schopenhauer. Der handschriftliche Nachlaß. Hrsg. von Arthur Hübscher, Bd. IV 2. Frankfurt am Main: Kramer, 1975: 131-267.

Gracian, Baltasar. The Art of Worldly Wisdom. A Pocket Oracle. Translated by Christopher Maurer. New York: Doubleday, 1992.

Gutzkow, Karl. „Arthur Schopenhauer's Lehre und Leben.“ In Karl Gutzkow, Unterhaltungen am häuslichen Her. Dritte Folge, 2. Band, 252-275. Leipzig: Heinrich Brockhaus Verlagsort, 1862.

Hoffmans, Gerd, Hrsg. Über Arthur Schopenhauer, 2. Aufl. Zürich: Diogenes, 1978. 
Hübscher, Arthur. Denker gegen den Strom. Schopenhauer: Gestern - Heute - Morgen. Bonn: Bouvier, 1973.

Lasierra, Juan Domínguez. „El año en que Gracián fue best-seller en los USA.” Turia. Revista cultural 54 (2000): 149-154.

Lindner, Ernst Otto. Arthur Schopenhauer. Von ihm. Ueber ihn. Ein Wort der Vertheidigung. Und Frauenstädt, Julius. Memorabilien, Briefe und Nachlassstücke. Berlin: A.W. Hayn, 1863.

Schirmacher, Wolfgang, Hrsg. Schopenhauers Aktualität. Ein Philosoph wird neu Gelesen. Wien: Passagen Verlag, 1988.

Schmidt, Alfred. Die Wahrheit im Gewande der Lüge. Schopenhauers Religionsphilosophie. München, Zürich: Piper, 1986.

Schopenhauer, Arthur. Der handschriftliche Nachlaß. Hrsg. von Arthur Hübscher, Bd. 1-5. Frankfurt am Main: Kramer, 1975.

Schopenhauer, Arthur. Gesammelte Briefe. Hrsg. von Arthur Hübscher, 2. Aufl. Bonn: Bouvier, 1987.

Schopenhauer, Arthur. Sämtliche Werke. Nach der ersten von Julius Frauenstädt besorgten Gesamtausgabe neu bearb. und hrsg. von Arthur Hübscher, 4. Aufl. durchges. von Angelika Hübscher. Mannheim: Brockhaus, 1988.

Schopenhauer, Arthur. Werke in fünf Bänden. Nach den Ausgaben letzter Hand. Hrsg. von Ludger Lütkehaus. Zürich: Hoffmans Verlag, 1994.

Schumm, Johanna. „Zur Wiederkehr der Verstellung: Die gegenwärtige Rezeption von Graciáns Oráculo manual als Ratgeber." Komparatistik Online 1 (2014): 204-228. https://www. komparatistik-online.de/index.php/komparatistik_online/article/view/132/94.

Spierling, Volker, Hrsg. Schopenhauer im Denken der Gegenwart: 23 Beiträge zu seiner Aktualität. München, Zürich: Piper, 1987.

\section{WISDOM IN SCHOPENHAUER'S GRASP AND THE QUESTION OF ITS TIMELINESS}

The notion of wisdom in Schopenhauer's grasp is analyzed in two different perspectives. First, in the context of early philosophy with the supreme term "better consciousness" (das bessere Bewußtsein). The crowning achievement of this period was The World as Will and Representation and radically formulated cognitive elitism. Second, in the context of Parerga and Paralipomena essays, in particular the Aphorisms on the Wisdom of Life essay, when Schopenhauer transforms himself from a metaphysician and a Buddhist into a moralist and teacher of life's wisdom, who teaches what to do to minimize the suffering of our lives. This shift of accents was connected with the thorough reading of B. Gracian's The Art of Worldly Wisdom. A Pocket Oracle. Gracian did not teach how to free oneself from the world, but how to live in a world full of evil, adversity, intrigues and wickedness.

\section{Keywords:}

Arthur Schopenhauer, Wisdom, Baltasar Gracian, German Philosophy 